\title{
Impiego del sistema Uro-Quick per l'identificazione di resistenze ben caratterizzate agli antibiotici veicolate da diverse specie batteriche
}

\author{
Simona Roveta, Anna Marchese, Eugenio A. Debbia
}

Università degli studi di Genova, Sezione di Microbiologia - DISCAT

The Uro-Quick system for the identification of well-characterized antibiotic-resistance strains.

Key Words: Uro-Quick, Detection of antibiotic resistance, Well-characterized strains

\section{SUMMARY}

The Uro-Quick system, previously used to carry out antibiotic susceptibility tests directly on urine samples, has been employed for the detection of antibiotic resistance traits that might be not easily detected in well-characterized bacterial species. The period of time requested by the instrument to identify the drug resistance were: penicillin-resistant (PEN-R), constitutive and inducible oxacillin-resistant (OXA-R) Staphylococcus aureus, OXA-R S. epidermidis 480 min. Teicoplanin-resistant (TEI-R) S. haemolyticus, Vancomycin- (VanA, VanB, VanC), aminoglycoside-resistant (HLAR) Enterococcus spp, 24h. S. pyogenes exhibiting the three erythromycin-resistance phenotypes (Ery-R, C, I e M) 480 min.Ampicillin- (AMP-R), ciprofloxacin (CIP-R) and clavulanic acid-resistant (IRT) Escherichia coli 180 min. Imipenem-resistant (IMI-R) Pseudomonas aeruginosa 24h. For each strain was evaluated the optimal experimental conditions including inoculum size, time of incubation and antibiotic concentration. The results obtained indicated that Uro-Quick might be employed for the correct detection of the above antibiotic resistant markers.

\section{INTRODUZIONE}

Lo strumento Uro-Quick rappresenta un sistema analitico automatico e dinamico che consente di superare i limiti dei classici sistemi turbidimetrici grazie all'utilizzo di misurazioni cinetiche della crescita batterica e della nefelometria a raggio laser (light scattering) che assicurano una sensibilità elevata. L'apparecchio provvede alla termostatazione $\left(37^{\circ} \mathrm{C}\right)$ e all'agitazione dei campioni, quest'ultima evita sedimentazioni, flottazioni ed anomalie di crescita, tipiche di alcuni microrganismi difficili. La tecnica nefelometrica light scattering utilizza un raggio di luce polarizzata che incide sui flaconcini contenenti un terreno di coltura liquido inoculato con i campioni da analizzare: i segnali in uscita che scaturiscono dalla cella di misura su cui incide il raggio luminoso vengono prelevati ed analizzati da due detector posti ad angoli di $30^{\circ} \mathrm{C}$ e $90^{\circ} \mathrm{C}$ rispetto alla luce incidente $(2,18)$. Il sistema Uro-Quick integra 5000 letture per ogni campione in un intervallo di tempo di 15 secondi al fine di "filtrare" i segnali ed ottenere un valore stabile. Lo strumento esegue le letture con cadenza di 5 minuti e registra i valori dei segnali in funzione del tempo. Il segnale ottico viene utilizzato in modo dinamico, registrando l'andamento nel tempo del segnale stesso ed interpretando tali variazioni per ottenere una valu- tazione del numero di microrganismi presenti. Al fine del calcolo tutta la curva segnale-tempo viene analizzata e rappresentata in forma matematica come funzione di 2 parametri, il numero iniziale di batteri e il loro tempo di duplicazione: lo strumento traccia quindi un grafico delle curve di crescita determinando la carica batterica iniziale.

Uro-Quick è utilizzato da tempo per la valutazione del numero di batteri presenti in un campione di urina, il sistema è così rapido che entro le 3 ore è possibile stimare la positività del campione $(8$, 19). In studi preliminari è stato accertato che il saggio di alcuni antibiotici può essere effettuato utilizzando questa metodica direttamente sui patogeni contenuti nel campione (14-15). In questo modo in un breve arco di tempo (6-8 ore) si possono ottenere informazioni sulla positività dell'urina e dell'eventuale sensibilità dei germi agli antibiotici più usati per il trattamento delle più comuni infezioni a carico di questo distretto anatomico.

In questo studio il sistema Uro-Quick è stato impiegato per la determinazione rapida della sensibilità agli antibiotici di ceppi Gram-negativi e Gram-positivi veicolanti resistenze agli antibiotici ben caratterizzate. Per ciascun microrganismo da sottoporre al saggio è stata determinata la concentrazione di antibiotico, la carica batterica e il 
tempo di incubazione da utilizzare in modo da ottenere la massima riproducibilità dei saggi.

\section{MATERIALI E METODI}

\section{Microrganismi}

I ceppi utilizzati in questo studio includevano stipiti di Staphylococcus aureus, distinti in: produttore di penicillinasi (PEN-R) (12) resistente all'oxacillina (OXA-R) a causa della espressione costitutiva del gene mecA e con fenotipo (OXAR, I) di tipo inducibile (5), S. epidermidis, OXAR (11), S. haemolyticus, teicoplanino-resistente (TEI-R) (11). Enterococcus faecalis, refrattario alla vancomicina e alla teicoplanina $(\operatorname{Van} A)(1)$, resistente alla vancomicina e sensibile alla teicoplanina $(\operatorname{Van} B)(1)$, insensibile ad alto livello agli aminoglicosidi (HLAR) (16), E. faecium ampicillino-resistente (AMP-R) (11) ed E. gallinarum caratterizzato da basso livello di refrattarietà alla vancomicina e sensibile alla teicoplanina $(\operatorname{Van} C)(1)$. Sono stati inoltre valutati $S$. pyogenes appartenenti al fenotipo Ery-R, C (resistenza costitutiva ad alto livello nei confronti di tutti i macrolidi e di clindamicina, codificato dal gene $\operatorname{erm} B$ ), a quello Ery-R, I (resistenza inducibile determinata da ermTR) e infine a Ery-R, M (resistenza a basso livello nei confronti di macrolidi a 14 e 15 atomi e sensibilità a quelli a 16 atomi nonché a clindamicina, conferita dal gene mefA) (3-4,17,20). Escherichia coli, resistente all'ampicillina (AMP-R) dovuta a produzione di penicillinasi (questo studio), AMP-R ma resistente agli inibitori delle $\beta$-lattamasi TEM (IRT) (9), produttore di $\beta$-lattamasi a spettro esteso (ESBL) (10) e resistente alla ciprofloxacina (CIP-R) (9). Pseudomonas aeruginosa resistente all'imipenem (IMI-R) (questo studio). Come ceppi di controllo sono stati impiegati $E$. coli ATCC 25922, E. coli ATCC 35218, per i saggi includenti un $\beta$-lattamico e un inibitore di $\beta$-lattamasi, $P$. aeruginosa ATCC 27853, S. aureus ATCC 25923, E. faecalis ATCC 29212.

\section{Antibiotici}

Le polveri di penicillina, oxacillina, amoxicillinaclavulanato, ceftazidime, imipenem, teicoplanina, vancomicina, gentamicina, streptomicina, eritromicina, clindamicina e ciprofloxacina, sono state ottenute da fonti commerciali. Le soluzioni madre sono state allestite secondo le istruzioni del fornitore e diluite nel terreno prescelto.

\section{Saggi di sensibilità agli antibiotici}

Le minime concentrazioni inibenti (MIC) sono state determinate con il metodo della microdiluizione in brodo Mueller Hinton (MH) contenente
$\mathrm{Ca}++\mathrm{e} \mathrm{Mg}++$ o mediante sistema $\mathrm{E}$ test. Il metodo Kirby-Bauer della diffusione da dischetto è stato utilizzato in alcuni saggi in parallelo alla valutazione della MIC per confermare ulteriormente il fenotipo di resistenza. I saggi sono stati condotti e interpretati seguendo le linee guida suggerite dal National Committee for Clinical Laboratory Standards, 2002 (13).

\section{Valutazione dei fenotipi di resistenza $\beta$-lat- tamasi in S. aureus}

La $\beta$-lattamasi in $S$. aureus è stata individuata mediante saggio dell'agar diffusione utilizzando dischetti di penicillina e amoxicillina-clavulanato (AUG), il ceppo ha dimostrato resistenza al capostipite dei $\beta$-lattamici e sensibilità all'associazione con l'inibitore dell'enzima.

\section{Oxacillino-resistenza in $\boldsymbol{S}$. aureus}

L'oxacillino-resistenza inducibile o costitutiva in $S$. aureus è stata messa in evidenza utilizzando il saggio dell'agar screening in duplicato su piastre di Mueller-Hinton agar con $6 \mathrm{mg} / \mathrm{L}$ di oxacillina, con e senza il $4 \%$ di $\mathrm{NaCl}$ e un inoculo di $5 \times 10^{5}$. La lettura è stata eseguita dopo 24 ore di incubazione. I ceppi sono stati mantenuti ad una temperatura non superiore ai $35.5^{\circ} \mathrm{C}$. In queste condizioni il ceppo inducibile è risultato sensibile al farmaco sul terreno privo di sale, mentre lo stipite costitutivo ha manifestato resistenza all'oxacillina su entrambi i terreni. S. epidermidis OXA-R è stato in grado di crescere alla presenza di oxacillina su terreno $\mathrm{MH}$ non contenente $\mathrm{NaCl}$ confermando il suo carattere.

\section{Eritromicino-resistenza in Streptococcus pyo- genes}

I tre principali fenotipi Ery-R di Streptococcus pyogenes sono stati identificati mediante tecnica del doppio disco. Sulla superficie di una piastra di Mueller-Hinton agar al 5\% di sangue di montone sono stati posizionati due dischetti (eritromicina [ERY] 15mg e clindamicina [CLI] $2 \mathrm{mg}$ ) ad una distanza di $1.5-2 \mathrm{~cm}$. Dopo incubazione per 24 ore l'assenza di una zona di inibizione la crescita attorno ad entrambi i dischi è stata interpretata come espressione del fenotipo $\mathrm{C}$, mentre un alone di inibizione intorno al dischetto di clindamicina ha indicato il fenotipo M. Infine il fenotipo inducibile è stato rivelato dalla deformazione dell'alone di inibizione intorno al dischetto di clindamicina nella zona adiacente a quello di eritromicina.

\section{Teicoplanino-resistenza in S. haemolyticus}

S. haemolyticus resistente alla teicoplanina è stato identificato mediante determinazione della minima concentrazione inibente adottando la tecnica 
della microdiluizione in brodo (NCCLS, 2002). Il test è stato letto dopo 24 ore di incubazione a $35.5^{\circ} \mathrm{C}$.

\section{Produzione di ESBL in $E$. coli}

La produzione di ESBL in E. coli è stata valutata mediante $E$ test utilizzando le apposite strisce per la valutazione di questo enzima.

\section{Alto livello di resistenza agli aminoglicosi- di negli enterococchi}

L'alto livello di resistenza agli aminoglicosidi negli enterococchi è stato valutato in piastre di BHIA contenenti $500 \mathrm{mg}$ di gentamicina (GEN) o $2000 \mathrm{mg}$ di streptomicina (STR). Per il controllo di qualità di questo test è stato impiegato $E$. faecalis ATCC 29212. Sono stati utilizzati inoculi di $10^{6} \mathrm{CFU}$ per spot. Dopo incubazione per 24 ore in aerobiosi le piastre sono state esaminate, la presenza di una o più colonie o un alone di crescita è stato considerato indice di resistenza. Quando il saggio con la streptomicina è risultato negativo è stato incubato nuovamente per altre 24 ore.

\section{Ampicillina-resistenza in E. faecium}

L'insensibilità all'ampicillina in E. faecium è stata determinata mediante antibiogramma.

\section{Resistenza ai glicopeptidi negli enterococchi} Per l'accertamento della refrattarietà ai glicopeptidi è stato utilizzato il metodo della microdiluizione in brodo.

\section{Fenotipi di ampicillino-resistenza in $E$. coli}

Il ceppo di $E$. coli AMP-R è un isolato clinico caratterizzato fenotipicamente con il metodo Kirby-Bauer sulla base della sola resistenza all'ampicillina e sensibilità all'amoxicillina-clavulanato. Il ceppo IRT dimostra resistenza sia all'ampicillina sia all'amoxicillina-clavulanato, tutti gli altri fenotipi di sensibilità o resistenza sono stati valutati mediante antibiogramma.

\section{Resistenza all'imipenem in $P$. aeruginosa}

$P$. aeruginosa IMI-R è un ceppo di isolamento clinico identificato fenotipicamente con il metodo Kirby-Bauer sulla base della resistenza all'imipenem.

\section{Sistema Uro-Quick}

Lo strumento è dotato di boccettine o cuvette di vetro ottico specifico per la lettura ai raggi laser che contengono un terreno ricco, formulato dal produttore dello strumento e denominato "eugonico" che risulta idoneo per lo sviluppo di tutte le specie batteriche isolabili dalle urine. Alcuni esperimenti preliminari condotti nel nostro labo- ratorio hanno dimostrato che $S$. pyogenes può crescere in modo soddisfacente in questo substrato. In alcuni saggi, la valutazione della sensibilità agli antibiotici è stata effettuata in Mueller-Hinton brodo sia privo sia con l'aggiunta di NaCL al $4 \%$ preparati specificamente dal fornitore. Le cuvette sono state quindi inoculate con il ceppo e inserite nello strumento. Quest'ultimo è collegato ad un computer. Un apposito programma, modificabile in alcuni parametri dall'operatore, gestisce la procedura. Al tempo stabilito i dati ottenuti appaiono sullo schermo sotto forma di grafico della curva di crescita, unitamente al numero di batteri iniziali, al tempo di lettura e ai dati di identificazione del campione. Il programma provvede automaticamente alla stampa dei risultati. Ciascun antibiotico da saggiare è stato aggiunto ai $2 \mathrm{ml}$ di terreno Mueller-Hinton contenuto nella cuvetta UroQuick. La concentrazione di antibiotico con la quale cimentare lo stipite in esame è stata calcolata sulla base dei breakpoints suggeriti dall'NCCLS (2002) in modo che il valore fosse tra quelli inclusi nell'intervallo di sensibilità intermedia. Queste concentrazioni sono quelle che hanno dimostrato la migliore riproducibilità dei dati tra quelle valutate in alcuni saggi preliminari. Una boccettina di brodo priva di antibiotico è stata seminata come controllo. L'inoculo del ceppo in esame presente in $0.5 \mathrm{ml}$ di terreno è stato aggiunto al contenitore dello strumento in modo da risultare come carica definitiva compresa tra $5 \times 10^{5}$ e $1 \times 10^{6} \mathrm{CFU} / \mathrm{mL}$, il volume finale di terreno nella cuvetta risultava $2.5 \mathrm{ml}$. La lettura è stata effettuata dopo un intervallo di tempo variabile in funzione delle caratteristiche del ceppo considerato. L'assenza di sviluppo della popolazione microbica (sensibilità) è stata registrata da parte dello strumento con una linea piatta, mentre una curva di crescita è stata assunta come indice della capacità del germe di proliferare nonostante la presenza del farmaco (resistenza).

\section{RISULTATI}

Nella tabella 1 sono riportati gli antibiotici utilizzati in questo studio, i relativi breakpoints suggeriti da NCCLS e la concentrazione finale di composto alla quale i microrganismi sono stati esposti nella cuvetta Uro-Quick. Nella tabella 2, invece, sono specificate le condizioni di studio utilizzate con lo strumento in particolare sono riportati il terreno utilizzato per la crescita dei microrganismi, i periodi di tempo ottimali per l'incubazione di ciascuna specie batterica e l'inoculo iniziale. Questi parametri sono stati adottati sulla base di esperimenti preliminari che hanno fornito la 
migliore riproducibilità dei risultati.

In $S$. aureus produttore di penicillinasi lo strumento Uro-Quick rivela la resistenza alla penicillina già dopo un'incubazione di 300 minuti, tuttavia la lettura dei risultati dopo 480 minuti evidenzia una curva di crescita del tutto paragonabile a quella dei controlli. Quando il germe è stato esposto all'associazione amoxicillina-clavulanato il sistema ha registrato la totale assenza di crescita. Per l'accertamento dell'oxacillinoresistenza nello $S$. aureus sono state allestite apposite cuvette Uro-Quick contenenti $\mathrm{MH}$ brodo addizionato di $\mathrm{NaCl} 4 \%$ e altrettante prive di sale. Uroquick ha identificato l'oxacillinoresistenza in $S$. aureus mecA costitutivo e $S$. aureus mecA inducibile in modo inequivocabile dopo 480 minuti di incubazione nel terreno contenente $\mathrm{NaCl}$ mentre l'assenza di sale nel terreno di coltura ha reso lo stipite mecA inducibile sensibile all'oxacillina. S. epidermidis OXA-R ha manifestato il proprio carattere di resistenza in maniera riproducibile dopo incubazione per 480 minuti in cuvette di Uro-Quick contenenti $\mathrm{MH}$ brodo privo di $\mathrm{NaCl}$. S. haemolyticus resistente alla teicoplanina è stato evidenziato dallo strumento dopo 24 ore di incubazione. Letture eseguite a intervalli di tempo minori hanno fornito risultati non sempre concordanti. Anche il terreno utilizzato per la crescita dei germi ha dimostrato una certa influenza sulla valutazione di questo carattere di resistenza. Quando i batteri sono stati coltivati in terreno eugonico la resistenza alla teicoplanina è sempre stata accertata in modo riproducibile dopo 24 ore di incubazione in Uro-Quick. Su questo particolare ceppo è stata saggiata anche la sensibilità alla vancomicina ottenendo sempre letture coerenti con il carattere veicolato dal germe. Il ceppo infatti, dopo 24 ore di incubazione, risultava sensibile a questo antibiotico.

Per quanto riguarda gli Enterococchi resistenti ai glicopeptidi, E. faecalis VanA, E. faecalis VanB, E. gallinarum VanC, la refrattarietà alla vancomicina e alla teicoplanina è stata identificata correttamente tramite il sistema Uro-Quick dopo incubazione delle cuvette per 24 ore. In particolare tutti i ceppi veicolanti le resistenze ai glicopeptidi sono stati seminati in doppio e contemporaneamente su terreni contenenti vancomicina e teicoplanina alla concentrazione di $10 \mu \mathrm{g} / \mathrm{ml}$. Come atteso, il ceppo E. faecalis VanA ha dimostrato resistenza sia alla vancomicina sia alla teicoplanina, mentre E. faecalis VanB ha evidenziato insensibilità alla sola vancomicina. E. gallinarum VanC infine è risultato resistente a basso livello alla vancomicina $(10 \mu \mathrm{g} / \mathrm{ml})$ ma non è stato in grado di tollerare concentrazioni più alte di farmaco $(20 \mu \mathrm{g} / \mathrm{ml})$ lo stipite ha confermato la sua

Tabella I. Breakpoints degli antibiotici utilizzati in questo studio e concentrazioni di farmaco alle quali $i$ microrganismi sono stati esposti nella cuvetta Uro-Quick

\begin{tabular}{lcc}
\hline ANTIBIOTICO & $\begin{array}{c}\text { BREAKPOINTS } \\
\mu \mathrm{G} / \mathrm{ML} *\end{array}$ & $\begin{array}{c}\text { CONCENTRAZIONE NELLA } \\
\text { CUVETTA URO-QUICK } \mu \mathrm{G} / \mathrm{ML}\end{array}$ \\
\hline Penicillina & $\leq 0.1$ & 0.2 \\
Ampicillina (Enterobacteriaceae) & $8-32$ & 10 \\
Ampicillina & $8-16$ & 10 \\
(Enterococcus spp.) & & 3 \\
Oxacillina & $2-4$ & \\
(S. aureus) & & 0.3 \\
Oxacillina & $0.25-0.5$ & \\
(SCN)** & & 15 \\
Amoxicillina/Clavulanato & $8-32$ & 20 \\
Ceftazidime & $8-32$ & 20 \\
Cefotaxime & $8-64$ & 7 \\
Imipenem & $4-16$ & 2 \\
Ciprofloxacina & $1-4$ & 0.5 \\
Eritromicina & $0.25-1$ & 0.5 \\
Clindamicina & $0.25-1$ & 1000 \\
Streptomicina & $<1000$ & 500 \\
Gentamicina & $<500$ & 10 \\
Teicoplanina & $8-32$ & 10 \\
Vancomicina*** & $4-32$ & \\
(Enterococcus spp.) & & 5 \\
Vancomicina & $\leq 4$ & \\
(Staphylococcus spp) & & \\
*NCCLS, 2002 & & \\
** SCN, Stafilococchi coagulasi-negativi & & \\
**In alcuni saggi è stata utilizzata la concentrazione di 20 & (vedi testo) \\
\end{tabular}


Tabella 2. Condizioni di studio con il sistema Uro-Quick per la valutazione del carattere di resistenza di ciascuna specie batterica SPECIE FENOTIPO TERRENO UTILIZZATO* INOCULO TEMPO DI CRESCITA

\begin{tabular}{|c|c|c|c|c|c|}
\hline & & & (CFU/ML) & INCUBAZIONE & REGISTRATA** \\
\hline S. aureus & PEN-R & $\mathrm{MH}+\mathrm{PEN} 0.2 \mu \mathrm{g} / \mathrm{ml}$ & $5 \times 10^{5}$ & 480 minuti & + \\
\hline S. aureus & PEN-R & $\mathrm{MH}+\mathrm{AUG} \mathrm{I} 5 \mu \mathrm{g} / \mathrm{ml}$ & $5 \times 10^{5}$ & 480 minuti & - \\
\hline S. aureus & OXA-R & $\mathrm{MH}+4 \% \mathrm{NaCl}$ OXA $3 \mu \mathrm{g} / \mathrm{ml}$ & $5 \times 10^{5}$ & 480 minuti & + \\
\hline S. aureus & OXA-R & $\mathrm{MH}+$ OXA $3 \mu \mathrm{g} / \mathrm{ml}$ & $5 \times 10^{5}$ & 480 minuti & + \\
\hline S. aureus & OXA-R, I & $\mathrm{MH}+4 \% \mathrm{NaCl}+\mathrm{OXA} 3 \mu \mathrm{g} / \mathrm{ml}$ & $5 \times 10^{5}$ & 480 minuti & + \\
\hline S. aureus & OXA-R, I & $\mathrm{MH}+$ OXA $0.3 \mu \mathrm{g} / \mathrm{ml}$ & $5 \times 10^{5}$ & 480 minuti & - \\
\hline S. epidermidis & OXA-R & $\mathrm{MH}+$ OXA $0.3 \mu \mathrm{g} / \mathrm{ml}$ & $1 \times 10^{6}$ & 480 minuti & + \\
\hline S. haemolyticus & TEI-R & EUG + TEI $10 \mu \mathrm{g} / \mathrm{ml}$ & $1 \times 10^{6}$ & 24 ore & + \\
\hline S. haemolyticus & TEI-R & EUG + VAN $5 \mu \mathrm{g} / \mathrm{ml}$ & $1 \times 10^{6}$ & 24 ore & - \\
\hline E. faecalis & VanA & EUG + TEl $10 \mu \mathrm{g} / \mathrm{ml}$ & $1 \times 10^{6}$ & 24 ore & + \\
\hline E. faecalis & VanA & $\mathrm{EUG}+\mathrm{VAN} 10 \mu \mathrm{g} / \mathrm{ml}$ & $1 \times 10^{6}$ & 24 ore & + \\
\hline E. faecium & VanB & EUG + TEl $10 \mu \mathrm{g} / \mathrm{ml}$ & $1 \times 10^{6}$ & 24 ore & - \\
\hline E. faecium & VanB & $\mathrm{EUG}+\mathrm{VAN} 10 \mu \mathrm{g} / \mathrm{ml}$ & $1 \times 10^{6}$ & 24 ore & + \\
\hline E. gallinarum & $\operatorname{VanC}$ & EUG + TEI I0 $\mu g / \mathrm{ml}$ & $1 \times 10^{6}$ & 24 ore & - \\
\hline E. gallinarum & VanC & EUG + VAN $10 \mu \mathrm{g} / \mathrm{ml}$ & $1 \times 10^{6}$ & 24 ore & + \\
\hline E. gallinarum & VanC & EUG + VAN $20 \mu \mathrm{g} / \mathrm{ml}$ & $1 \times 10^{6}$ & 24 ore & - \\
\hline E. faecalis & HLAR & EUG + STR $1000 \mu \mathrm{g} / \mathrm{ml}$ & $1 \times 10^{6}$ & 24 ore & + \\
\hline E. faecalis & HLAR & EUG + GEN $500 \mu \mathrm{g} / \mathrm{ml}$ & $1 \times 10^{6}$ & 24 ore & + \\
\hline E. faecium & AMP-R & $\mathrm{MH}+\mathrm{AMP} 10 \mu \mathrm{g} / \mathrm{ml}$ & $1 \times 10^{6}$ & 480 minuti & + \\
\hline S. pyogenes & Ery-S & EUG & $1 \times 10^{6}$ & 480 minuti & + \\
\hline S. pyogenes & Ery-S & $\mathrm{EUG}+\mathrm{ERY} 0.5 \mu \mathrm{g} / \mathrm{ml}$ & $1 \times 10^{6}$ & 480 minuti & - \\
\hline S. pyogenes & Ery-R, C & $\mathrm{EUG}+\mathrm{ERY} 0.5 \mu \mathrm{g} / \mathrm{ml}$ & $1 \times 10^{6}$ & 480 minuti & + \\
\hline S. pyogenes & Ery-R, C & $\mathrm{EUG}+\mathrm{CLI} 0.5 \mu \mathrm{g} / \mathrm{ml}$ & $1 \times 10^{6}$ & 480 minuti & + \\
\hline S. pyogenes & Ery-R, M & EUG + ERY $0.5 \mu \mathrm{g} / \mathrm{ml}$ & $1 \times 10^{6}$ & 480 minuti & + \\
\hline S. pyogenes & Ery-R, M & EUG + CLI $0.5 \mu \mathrm{g} / \mathrm{ml}$ & $1 \times 10^{6}$ & 480 minuti & - \\
\hline S. pyogenes & Ery-R, M & EUG + CLI $0.5+$ ERY $0.5 \mu \mathrm{g} / \mathrm{ml}$ & $1 \times 10^{6}$ & 480 minuti & - \\
\hline S. pyogenes & Ery-R, I & EUG + ERY $0.5 \mu \mathrm{g} / \mathrm{ml}$ & $1 \times 10^{6}$ & 480 minuti & + \\
\hline S. pyogenes & Ery-R, I & $\mathrm{EUG}+\mathrm{CLI} 0.5 \mathrm{~g} / \mathrm{ml}$ & $1 \times 10^{6}$ & 480 minuti & - \\
\hline S. pyogenes & Ery-R, I & EUG + CLI $0.5+$ ERY $0.5 \mu \mathrm{g} / \mathrm{ml}$ & $1 \times 10^{6}$ & 480 minuti & + \\
\hline E. coli & AMP-R & $\mathrm{MH}+\mathrm{AMP} 10 \mu \mathrm{g} / \mathrm{ml}$ & $5 \times 10^{5}$ & 180 minuti & + \\
\hline E. coli & AMP-R & $\mathrm{MH}+\mathrm{AUG}$ I5 $\mu \mathrm{g} / \mathrm{ml}$ & $5 \times 10^{5}$ & 180 minuti & - \\
\hline E. coli & CIP-R & $\mathrm{MH}+\mathrm{CIP} 2 \mu \mathrm{g} / \mathrm{ml}$ & $5 \times 10^{5}$ & 180 minuti & + \\
\hline E. coli & AMP-R, IRT & $\mathrm{MH}+\mathrm{AMP} 10 \mu \mathrm{g} / \mathrm{ml}$ & $5 \times 10^{5}$ & 180 minuti & + \\
\hline E. coli & AMP-R, IRT & $\mathrm{MH}+\mathrm{AMP} 10+\mathrm{AUG} \mathrm{I} 5 \mu \mathrm{g} / \mathrm{ml}$ & $5 \times 10^{5}$ & 180 minuti & + \\
\hline E. coli & ESBL & $\mathrm{MH}+\mathrm{CAZ} 20 \mu \mathrm{g} / \mathrm{ml}$ & $5 \times 10^{5}$ & 180 minuti & + \\
\hline E. coli & ESBL & $\mathrm{MH}+\mathrm{CTX} 20 \mu \mathrm{g} / \mathrm{ml}$ & $5 \times 10^{5}$ & 180 minuti & + \\
\hline E. coli & ESBL & $\mathrm{MH}+\mathrm{CAZ} 20+\mathrm{AUG} 15 \mu \mathrm{g} / \mathrm{ml}$ & $5 \times 10^{5}$ & 180 minuti & - \\
\hline E. coli & ESBL & $\mathrm{MH}+\mathrm{CTX} 20+\mathrm{AUG} 15 \mu \mathrm{g} / \mathrm{ml}$ & $5 \times 10^{5}$ & 180 minuti & - \\
\hline E. coli & ESBL & $\mathrm{MH}+\mathrm{IMI} 7 \mu \mathrm{g} / \mathrm{ml}$ & $5 \times 10^{5}$ & 180 minuti & - \\
\hline P. aeruginosa & IMI-R & $\mathrm{MH}+\mathrm{IMI} 7 \mu \mathrm{g} / \mathrm{ml}$ & $5 \times 10^{5}$ & 24 ore & + \\
\hline
\end{tabular}

*MH Mueller-Hinton; EUG eugonico; ** + crescita; - assenza di crescita; Vedi testo per ulteriori dettagli e abbreviazioni

sensibilità alla teicoplanina.

E. faecalis HLAR, caratterizzato da alti livelli di resistenza agli aminoglicosidi, è stato saggiato per streptomicina e gentamicina alle concentrazioni di $1000 \mu \mathrm{g} / \mathrm{mL}$ e $500 \mu \mathrm{g} / \mathrm{mL}$ rispettivamente. Questo ceppo ha richiesto un'incubazione di 24 ore in terreno eugonico perché la resistenza venisse correttamente rilevata dal Sistema Uro-Quick. Letture eseguite a intervalli di tempo minori (6-8 ore) hanno fornito comunque risultati concordanti in circa $1^{\prime} 80 \%$ dei casi.

Per il saggio con il sistema Uro-Quick di E. faecium ampicillino-resistente la curva di crescita ottimale del ceppo in presenza di ampicillina è stata ottenuta dopo un'incubazione di 480 minuti in terreno Mueller-Hinton.

Per i ceppi di S. pyogenes resistenti all'eritromicina sono stati adottati metodi di accertamento che hanno comportato il saggio contemporaneo all'eritromicina, alla clindamicina da sola o in presenza di $0.5 \mathrm{mg} / \mathrm{L}$ del capostipite dei macrolidi come induttore di resistenza. In particolare il fenotipo Ery- $\mathrm{R}, \mathrm{C}$ ha dimostrato crescita in entrambi i terreni contenenti i due distinti antibiotici indipendentemente dalla presenza del composto induttore. Lo stipite di tipo Ery-R, M ha evidenziato resistenza all'eritromicina e sensibilità alla clindamicina anche quando quest'ultima è stata combinata con eritromicina come induttore. Il ceppo con resistenza inducibile all'eritromicina Ery-R, I è 
risultato capace di crescere sia in presenza di eritromicina sia in presenza di clindamicina solo se quest'ultima è stata associata al macrolide induttore a 14 atomi.

Per quanto riguarda $E$. coli sia ampicillino-resistente sia refrattario alla ciprofloxacina il sistema Uro-Quick ha identificato tali caratteri in 180 minuti con i ceppi coltivati in Mueller-Hinton. $E$. coli AMP-R, IRT resistente agli inibitori delle $\beta$-lattamasi ha dimostrato crescita in terreno contenente ampicillina e l'associazione amoxicillina/clavulanato dopo 180 minuti. Infine E. coli produttore di ESBL è risultato resistente a ceftazidime (CAZ) e cefotaxime (CTX). Quando queste ultime cefalosporine sono state associate con amoxicillina-clavulanato o il ceppo è stato cimentato con imipenem il sistema Uro-Quick ha registrato totale assenza di crescita batterica dopo incubazione di 180 minuti in terreno MuellerHinton.

$P$. aeruginosa resistente a imipenem ha manifestato il proprio carattere di refrattarietà dopo 24 ore di incubazione nello strumento Uro-Quick.

\section{CONCLUSIONI}

Rispetto alle metodiche tradizionali, i sistemi automatizzati o semiautomatici presentano alcuni fattori critici che più di altri possono influire sul corretto accertamento della resistenza agli antibiotici, tra cui l'inoculo, la specie batterica considerata, la temperatura e il tempo di incubazione, nonché la concentrazione di farmaco alle quali esporre i vari microrganismi.

La valutazione mediante Sistema Uro-Quick delle sensibilità agli antibiotici sia sui ceppi Grampositivi che su quelli Gram-negativi, quando condotta in condizioni ottimali, come quelle ritrovate qui per le singole combinazioni antibiotico-specie batterica, ha dimostrato totale concordanza con i dati ottenuti utilizzando i metodi tradizionali (Kirby-Bauer, E test). Sperimentando alcune variazioni sul tempo di incubazione e sulla concentrazione finale di antibiotico nella cuvetta Uro-Quick è stato infatti possibile incrementare la riproducibilità dei dati fino al $100 \%$. Studi precedenti avevano già mostrato su saggi condotti direttamente su campioni di urina (senza essere quindi a priori a conoscenza del patogeno coinvolto) concordanze superiori al $90 \%(14,15)$.

Alla luce dei risultati ottenuti sono possibili le seguenti considerazioni: il sistema appare duttile, rapido e programmabile sulla base del patogeno e dell'antibiotico considerato. I ceppi qui studiati non includono tutti i possibili fenotipi ritrovabili nella diagnostica, ma rappresentano un campione significativo di quelli che si incontrano in ambiente ospedaliero. L'elenco richiede un aggiornamento ed un ampliamento proprio per includere quelle situazioni di resistenza non ancora considerate e per incrementare le informazioni preliminari qui raccolte. Il terreno eugonico qui affiancato al classico Mueller-Hinton risponde bene alle esigenze nutrizionali di molte specie e non appare incidere sulla valutazione di molti fenotipi di resistenza.

Come per altri metodi automatici o semiautomatici alcuni parametri sono risultati fondamentali per l'identificazione dei caratteri di resistenza (6-7), l'inoculo e il tempo di incubazione. Uro-Quick che stima la capacità dei microrganismi di proliferare da valori anche bassi di concentrazione batterica, non evidenziabili dai sistemi ottici tradizionali, può offrire in alcuni casi il vantaggio di una lettura che anticipa il comportamento dei microrganismi rispetto a metodi più tradizionali con un buon guadagno di tempo.

\section{BIBLIOGRAFIA}

1. Boisivon A, Thibault M, Leclercq R. Colonization by vancomycin-resistant enterococci of the intestinal tract of patients in intensive care units from French general hospitals. Clin Microbiol Infect 1997; 3: 175-9.

2. Breda E. Principles and technology of the Uro-Quick system for bacteriuria rapid screening. Galeno1996; 4: 11-21.

3. Chelossi E, Platè M, De Leo C, Schito GC. Attività di alcuni antibiotici su Streptococcus pyogenes eritromicino-resistenti isolati recentemente in Italia. GIMMOC, 1997; 3: 9-20.

4. Chelossi E, Platè M, DeLeo C, Schito GC. Analisi genotipica di Streptococcus pyogenes eritromicinoresistenti isolati recentemente in Italia GIMMOC 1998; 2: 9-20.

5. Chung $\mathrm{M}$, de Lencastre $\mathrm{H}$, Matthews $\mathrm{P}$, et al. Molecular typing of methicillin-resistant Staphylococcus aureus by pulsed-field gel electrophoresis: comparison of results obtained in a multilaboratory effort using identical protocols and MRSA strains. Microb Drug Resist 2000; 6: 189-98.

6. Felmingham D, Brown DFJ. Instrumentation in antimicrbial susceptibility testing. J Antimicrob Chemother 2001; 48 (suppl S1): 81-5.

7. Ferraro MJ, Jorgensen JH. Susceptibility testing instrumentation and computerized expert systems for data analysis and interpretation. Murray PR, et al.: Manual of Clinical Microbiology, Seventh Edition, ASM Press 1999: 1593-600.

8. Iverson D, Fayette R, Johnson C, Shigei J, Peterson E, Pezzlo M. Detection of bacteriuria by a rapid, three hours automated screening method. Abstr C-39, ASM. Chicago, USA, 1999.

9. Livermore D, Struelens M, Amorin J, et al. Multicentre evaluation of the VITEK 2 advanced export system for interpretative reading of antimicrobial resistance tests. J Antimicrob Chemother 2002; 49: 289-300.

10. Marchese A, Arlet G, Schito GC, Lagrange PH, Philippon A. Detection of SHV-5 extended-spectrum $\beta$-lactamase in Klebsiella pneumoniae strains isolated in Italy. Eur J Clin Microbiol Infect Dis 1996; 15: 
245-8.

11. Marchese A, Debbia EA, Bacca D, Balistreri G, Musolino B, Schito GC. Multidrug-resistant Grampositive pathogens: an update on current microbiological patterns. Drugs 1997; 54: Suppl 6, 11-20.

12. Marchese A, Saverino D, Debbia EA, Pesce A, Schito GC. Antistaphylococcal activity of cefdinir, a new oral third generation cephalosporin, alone and in combination with other antibiotics, at supra- and subMIC levels. J Antimicrob Chemother 1995; 35: 5366.

13. National Committee for Clinical Laboratory Standards. Performance standards for antimicrobial susceptibility testing. Twelfth informational supplement: M100-S12; Vol. 22. NCCLS, Wayne, PA. 2002.

14. Roveta S, Debbia EA, Marchese A. Valutazione della sensibilità agli antibiotici di uropatogeni mediante sistema Uro-Quick direttamente sul campione di urine e confronto con il metodo Kirby-Bauer. GIMMOC, 2003, in stampa.

15. Roveta S, Balistreri MR, Marchese A, Debbia EA. Sistema rapido Uro-Quick per l'esecuzione di antibiogrammi: valutazione comparativa con il sistema Kirby-Bauer. Abstr 36; 300. Congr Naz Soc It Microbiol Catania, 2002.

16. Schito GC, Molinari G, Debbia EA, Shaw KJ, Sabatelli F, Hare RS, Miller GH. Mechanisms of aminoglycoside resistance in a large italian hospital. Abstr A-67, 90th Annual Meeting ASM, 1990.

17. Seppälä H, Nissinen A, Yu Q, Huovinen P. Three different phenotypes of erythromycin-resistant Streptococcus pyogenes in Finland. J Antimicrob Chemother 1993; 32: 885-91.

18. Sonak R, Thoholte H. Determination of the number of pathogens in bacterial suspection using laser nephelometry in comparison to the photometry and plate counting method. Zentralbl. Bakteriol Mikrobiol Hyg 1985; 260: 329-38.

19. Soro O, Schito GC, Raggi M. Performance of a new automated method for the detection of bacteriuria. Abstr 1598, $7^{\text {th }}$ ECCMID, Vienna, Austria, 1995.

20. Sutcliffe J, Tait-kamradt A, Wondrack L. Streptococcus pneumoniae and Streptococcus pyogenes resistant to macrolides but sensitive to clindamycin: a common resistance pattern mediated by an efflux system. Antimicrob. Agents Chemoter 1996; 40: 1817-24.

\section{Eugenio A. Debbia \\ Università degli Studi di Genova DISCAT - Sez. Microbiologia- L.go Rosanna Benzi 10 - 16132 Genova Tel.: 010 3537655; Fax 010504837 e-mail: eugenio.debbia@unige.it}

